\section{HIV defense}

Soluble factors secreted by $\mathrm{CD} 8^{+} \mathrm{T}$ cells can inhibit HIV replication. $\beta$-chemokines (RANTES, MIP- $1 \alpha$ and MIP-1 $\beta$ ) block infection by HIV strains that use CCR5 ("R5" isolates), but the identity of the CD8 antiviral factor (CAF) that inhibits both R5 and "X4" (viruses that use CXCR4) isolates has remained elusive. In Science, Ho and colleagues describe how they used mass spectroscopy and protein-chip technology to isolate three proteins that are secreted in response to stimulation of $\mathrm{CD} 8^{+} \mathrm{T}$ cells from HIV-infected long-term nonprogressors. These proteins were identified as $\alpha$ defensins-1, -2 and -3 . Blocking antibody for $\alpha$-defensins abolished anti-HIV activity not attributable to $\beta$-chemokines. Furthermore, synthetic and purified human $\alpha$-defensins inhibited HIV replication in vitro. Thus, $\alpha$ defensins contribute to the anti-HIV activity of CAF.

Science, 26 Sept 2002 (doi:10.1 I 26/science. 1076185)

\section{Pointed autoimmunity}

The IL-6 receptor consists of an $\alpha$ chain paired with the subunit gp130. By introducing a single point mutation of gp130 (Y759 $\rightarrow$ F759), Hirano and colleagues generated SHP-2 signal-deficient mice. They report in the Journal of Experimental Medicine that these mice spontaneously develop rheumatoid arthritis. Mutant mice produce autoantibodies and have greater numbers of activated $\mathrm{T}$ cells and myeloid cells. Both thymic selection and clonal deletion of peripheral $\mathrm{T}$ cells were impaired. Mature T cells exhibited hyperresponsiveness even before disease onset, and IL-6-induced STAT3 activation was prolonged, which enhanced the inhibitory effect of IL-6 on FasL expression. Thus, a point mutation in a cytokine receptor can effect B and T cell abnormalities that cause autoimmunity. These mice may provide a useful animal model for spontaneous rheumatoid arthritis.

J. Exp. Med. 196, 979-990

\section{STAT2 recruits BRG I}

IFNs induce antiproliferative and antiviral cellular responses by signaling though the transcriptional activator STAT proteins, leading to de novo transcription of IFN-regulated genes. In Nature Cell Biology, Huang et al. show cells activated by IFN- $\alpha$ translocate STAT2 to the nucleus, where it interacts with BRG1, an ATP-dependent chromatin-remodeling protein. STAT2 recruits BRG1 to some, but not all, IFN- $\alpha-$ responsive promoters. IFN-responsiveness of these genes requires chromatin structural changes, as ATPase-defective BRG1 mutant cells could not activate the appropriate promoters. These results suggest IFN- $\alpha$-inducible genes have differential requirements for their expression in addition to binding the transactivator STAT2. How BRG1 alters the chromatin environment of IFN- $\alpha$-responsive genes remains to be shown.

Nature Cell Biol. 4, 774-78I (2002)

\section{Anaphylatoxin-responsive DCs}

A distinct population of DCs exist in the bloodstream that can respond to the anaphylatoxins $\mathrm{C} 3 \mathrm{a}$ and $\mathrm{C} 5 \mathrm{a}$ and induce potent antibacterial responses. In Immunity, Rieber and colleagues identify the carbohydrate 6-sulfo LacNAc, which modifies the ligand for Pselectin (PSGL-1), as a marker for this DC subset. This compound prevents the DCs from binding to both P- and E-selectins; instead, these cells express C3aR, C5aR and CD16 (an activating $\mathrm{Fc} \gamma$ receptor), which promote DC recruitment to inflammatory sites. Upon lipopolysaccharide, but not viral, activation, these DCs secrete vast amounts of TNF- $\alpha$ and display potent $\mathrm{T}$ cell priming activities. Thus, this 6-sulfo $\mathrm{LacNAc}^{+} \mathrm{DC}$ subset may represent the major proinflammatory APCs that respond to septic bacterial infections.

Immunity I7, 289-30I (2002)

\section{Rapamycin kills via p27 ${ }^{\text {kipl }}$}

Rapamycin is an immunosuppressant used commonly to prevent allograft rejection. Although its effects on $\mathrm{T}$ cells are well known, rapamycin may also affect other cells. In Blood, Woltman et al. show that rapamycin, through its target the lipid kinase mTOR, can specifically induce DC apoptosis by interfering with survival pathways mediated by GM-CSF. DC survival appears to be exquisitely sensitive to inhibitors of both PI3K and mTOR. Blocking either mTOR activity or growth factor withdrawal leads to the up-regulation of cell cycle inhibitor $\mathrm{p} 27^{k i p l}$, which, in turn, can down-regulate the anti-apoptotic Bcl2-related protein Mcl-1. Loss of Mcl-1 expression in DCs is sufficient to trigger apoptosis. Thus, in addition to rapamycin's known effects, it also inhibits immune responses by inducing DC apoptosis.

Blood, 26 Sept 2002 (doi:10.1 I82/blood-2002-06-1688)

\section{Downstream divergence}

The MyD88 signaling pathway is shared by all TLRs. However, TLR3 and TLR4 can activate the transcription factors NF- $\mathrm{\kappa B}$ and IRF3 in a MyD88-independent manner. Although activation of the NF- $\kappa$ B pathway leading to inflammatory responses is well characterized, the functional role of IRF3 is not understood. In Immunity, Doyle et al. show that activation of both TLR3 and TLR4 results in the specific induction of a set of five "IFN-regulated" genes through IRF3. Among the products of these genes, IFN- $\beta$ further induces other genes that are important in viral immunity. When compared to other TLRs, activation of cells through TLR3 and TLR4 results in the most potent resistance to viral replication. Thus, TLR3 and TLR4 have evolved to mediate innate antiviral responses via IRF3 activation.

Immunity I7, 25I-263 (2002)

\section{Different ways to die}

The Bcl-2 protein family is thought to exert its anti-apoptotic effects by blocking the release of cytochrome $\mathrm{c}$ from the mitochondria and thus prevents the activation of the apoptosome. However, the mechanism of action of Bcl-2 remains controversial because it prevents death independently of mitochondrial factors in C. elegans. In Nature, Marsden et al. show that Bcl-2 can regulate the activation of initiator caspases independently of the "apoptosome". Whereas the overexpression of Bcl-2 inhibits cell death mediated by various stimuli, the absence of apoptosome components Apaf-1 and caspase-9 does not delay cell death. Caspase activity is detected in the absence of apoptosome and is likely to be mediated by caspase- 7 . Thus, there is an alternate initiator caspase pathway, which is regulated by Bcl-2.

Nature, 25 September 2002 (doi: I0.1038/nature0I I0I) 